\title{
Health is wealth and share the wealth of health
}

\author{
Dr. K.R.P. Chapman \\ Consultant Surgeon, Chilaw
}

Dr. S.M.K.D. Arunatileka

sUniversity of Colombo School of Computing, Sri Lanka

Dr. K.T. Wickramasuriya

University of Colombo School of Computing, Sri Lanka

C. Ranatunga

Skynet Solutions

Contact e-mail address: shiromi_a@hotmail.com

eHealth Sri Lanka 2010,1(suppl.1):S36

DOI: http://dx.doi.org/10.4038/sljbmi.v1i0.3599

Only the Abstract is available

\begin{abstract}
Knowledge transfers among medical professionals are limited to lectures in the educational institutes in Sri Lanka. This is a limited resource restricted to educational institutions and city based hospitals in Sri Lanka. 'saukya.lk' is an exclusive social networking service with state of the art technology for consultants, doctors, medical students and other professionals. The system is dedicated to provide top class health information and manage social network with knowledge. It helps to establish and maintain personal and professional relationships between people from all over the world. 'saukya.lk' will address these issues by providing the facility to allow users to share their knowledge and experiences. 'saukya.lk' helps users to gain medical knowledge, Find health related information, find people from all over the world, establish new personnel or professional connection, discuss medical cases, share your personnel knowledge, experiences and ideas, review the latest drugs and medical equipment in the market and many more. It is the first ever online medical health forum for healthcare professionals in Sri Lanka.

The main functionalities and features that covered in the 'Saukya.lk' are separate medical forums for authors/readers according to their privileges, facility to share videos, audios, images, test results (non-confidential) chat facility, possibility to add medical articles to the system, information about conferences, scholarships, medical courses, new medical equipment, most common diseases in the society, health sector in Sri Lanka and in South Asia. It also provides information about Ministry of Healthcare and Nutrition, Medical Schools, Medical Associations, etc. 'saukay.lk' has several medical resources such as journal papers, journals, research papers, conference papers, medical dictionary, CDs, e-books, etc. Health is a primary human right and 'saukya.lk' will be a bridge to connect consultants, doctors and students in a more interactive manner proving that health is wealth and will share the wealth of health.
\end{abstract}

Keywords - health is wealth, wealth of health, saukya.lk 